\title{
Investigation of the presence of Trichomonas vaginalis in infertile Turkish women
}

\author{
Özlem Aycan Kaya ${ }^{a}$, Dilek Benk Silfeler ${ }^{b}$, Raziye Keskin Kurtc, Ilay Gö z ükara ${ }^{\mathrm{d}}$, Erhan Yengile, Neslihan \\ Bayramog̈lu \\ ${ }^{a}$ Mustafa Kemal University, Faculty of Medicine, Department of Parasitology, Hatay 31100, Turkey \\ ${ }^{b}$ Mustafa Kemal University, Faculty of Medicine, Department of Obstetrics and Gynecology, Hatay \\ 31100, Turkey \\ 'Mustafa Kemal University, Faculty of Medicine, Department of Family Medicine, Hatay 31100, Turkey
}

\begin{abstract}
Background: Trichomoniasis is a sexually transmitted vaginal infection caused by a protozoan called Trichomonas vaginalis. Its prevalence ranges between 3-40\% among randomly tested women .

Objectives: To investigate the presence of T. vaginalis in infertile women presenting to a Turkish gynecology outpatient clinic using parasitological methods.

Methods: The study population comprised 51 patient participants who attended the gynecology clinic of Mustafa Kemal University Hospital between March and August 2013. The women were divided into those presenting with infertility $(n=22)$ and a patient control group $(n=29)$. Women in the control group had complaints other than infertility. Microscopic examination, Giemsa staining, and cysteine-peptone-liver-maltose culture were performed on samples taken from posterior fornix tested for T. vaginalis.

Results: T. vaginalis was observed in $18 \%(n=4)$ of infertile patients and in none of the control group $(P=0.03)$. Conclusions: Asymptomatic infertile women should be examined for T. vaginalis infection, which may play a role as a cause or contributing factor in infertility.
\end{abstract}

Keywords: Infertility, parasitological diagnosis, Trichomonas vaginalis

Trichomoniasis is a common sexually transmitted parasitic disease [1]. Its prevalence is usually higher than for other sexually transmitted diseases (STDs). Diagnosis of Trichomonas vaginalis infection is cumbersome, and the infection is often asymptomatic and underdiagnosed. Trichomoniasis is an STD that increases the risk of human immunodeficiency virus (HIV) infection [2]. Asymptomatic trichomoniasis is associated with pregnancy complications such as premature rupture of membranes or amniorrhexis, preterm labor, low birth weight, endometritis after cesarean section, and abortus. Furthermore, newborn girls may be infected during birth and their infection may remain silent [3]. Trichomoniasis was found to be related to infertility and cervical dysplasia [4]. T. vaginalis infection, though often asymptomatic, may also result in a broad spectrum of signs and

Correspondence to: Özlem Aycan Kaya, Department of Parasitology, Faculty of Medicine, Mustafa Kemal University, Hatay 31100, Turkey. E-mail: omkaya@mku.edu.tr symptoms, such as foul smelling vaginal discharge, pelvic pain, dyspareunia, and dysuria. Furthermore, T. vaginalis infection may cause infertility because of endometritis, salpingitis, and atypical pelvic inflammatory symptoms $[5,6]$.

There is no simple diagnostic method with adequate sensitivity and specificity for $T$. vaginalis infection [1]. Therefore, special diagnostic tests are needed to confirm the presence of $T$. vaginalis. Diagnosis is usually made via observing parasites in a wet mount preparation. Although this method is inexpensive and fast, sensitivity is not high and the parasite is detected in only $60 \%$ of culture positive samples. Novel diagnostic methods such as nucleic acid amplification are more sensitive than routine diagnostic methods, but more expensive and not generally available $[7,8]$.

The aim of this study was to compare the presence of $T$. vaginalis in infertile and fertile Turkish women and to investigate the association of $T$. vaginalis with infertility. 


\section{Materials and methods}

The ethics committee of Zeynep Kamil Women's and Children's Hospital, approved the study (protocol number 30.09.2013/93) and written informed consent was obtained from all patient participants .

The study population comprised 51 participants applying to the gynecology outpatient clinic between March and August 2013. The participants were divided into 2 groups: an infertility group $(\mathrm{n}=22)$ and control group $(\mathrm{n}=29)$. Women in the control group had complaints other than infertility. Women having systemic disease, pregnancy, foul smelling vaginal discharge, and taking antibiotics were excluded from study. Routine gynecological examinations were performed after women were informed about the objectives of the examination and a signed consent form was obtained. Three samples were taken from the posterior fornix via sterile cotton swabs during a speculum examination. The first sample was mixed with $1 \mathrm{~mL}$ sterile saline and direct microscopy was performed. One drop from the mixture was put on a slide and examined using light microscopy at $\times 400$ magnification. The second sample was examined using light microscopy after Giemsa staining. The third sample was cultured in tubes containing cysteinepeptone-liver-maltose medium (CPLM). The tubes were incubated at $37^{\circ} \mathrm{C}$ for 48 hours and examined.

In both groups, after $12 \mathrm{~h}$ fasting on the third day of the menstrual cycle, the hormone profile or the patient and complete blood counts were measured.
Hormone profile included follicle stimulating hormone (FSH), luteinizing hormone (LH), and $17 \beta$-estradiol (E2). These hormones were measured using standard enzymatic methods with a fully automated random access chemiluminescence-enhanced enzyme immunoassay system (Roche Laboratory Systems, Mannheim, Germany).

The data obtained were analyzed using PASW Statistics for Windows, version 18.0 (SPSS Inc, Chicago, IL, USA). The relationship between categorical variables in groups was evaluated using a chi-square or Fisher's exact test. The relationship between continuous variables in groups was evaluated using a Student $t$ test. $P<0.05$ is was considered significant.

\section{Results}

T. vaginalis testing was positive in $18 \%(n=4)$ of women in the infertile group and $T$. vaginalis was not observed in the control group (Figure 1).

There was a significant difference in prevalence of $T$. vaginalis between the groups ( $P=0.03$ (odds ratio: 2.6 (95\% confidence interval 1.8 to 3.7))).

The mean age, hemoglobin, hematocrit, platelet counts, FSH, LH, and E2 levels of all participants are presented in Table 1. Reasons of infertility are shown in Table 2.

While three patients with $T$ vaginalis had unexplained infertility, one patient infected with $T$ vaginalis also had male factor infertility.

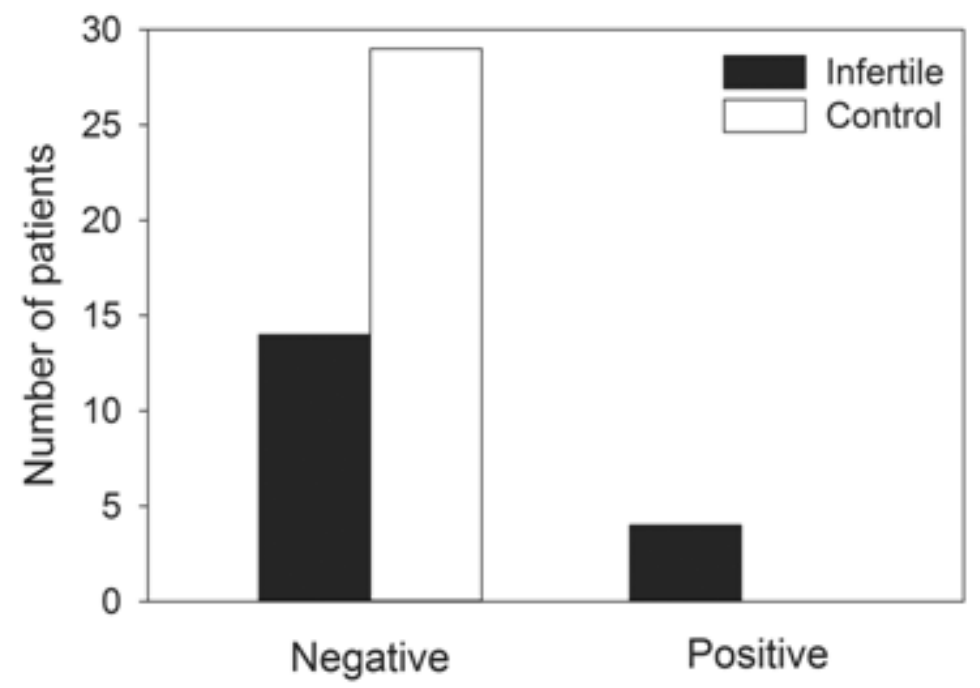

Figure 1. Incidence of Trichomonas vaginalis in infertile and control patient groups 
Table 1. Relationship of continuous variables in between groups

\begin{tabular}{llll}
\hline & Infertility & Control & $\boldsymbol{P}^{\mathbf{a}}$ \\
\hline Age (years) & $29.5 \pm 4.63$ & $32.7 \pm 6.45$ & 0.06 \\
Hemoglobin (g/dL) & $12.09 \pm 1.50$ & $12.45 \pm 1.48$ & 0.40 \\
Hematocrit (\%) & $35.87 \pm 4.67$ & $37.20 \pm 4.63$ & 0.32 \\
Platelets $\left(\times 1000 / \mathrm{mm}^{3}\right)$ & $301.59 \pm 136.34$ & $242.51 \pm 65.63$ & 0.046 \\
FSH(IU/L) & $14.52 \pm 17.41$ & $10.18 \pm 8.75$ & 0.25 \\
LH(IU/L) & $7.64 \pm 5.97$ & $8.88 \pm 7.88$ & 0.54 \\
Estradiol (pmol/L) & $105.92 \pm 175.92$ & $89.35 \pm 87.44$ & 0.66 \\
\hline
\end{tabular}

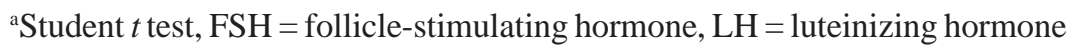

Table 2. Causes of infertility in infertile group

\begin{tabular}{lcc}
\hline & Frequency & Percent \\
\hline Unexplained & 7 & 31 \\
Female factor (tubal factor and low ovarian reserve) & 8 & 36 \\
Male factor & 7 & 31 \\
\hline
\end{tabular}

\section{Discussion}

Trichomonas vaginalis infection results from an anaerobic flagellated protozoan and may result in serious health consequences, but is readily treatable [9]. This infection has been related to vaginitis, endometritis, salpingitis, infertility, preterm labor, low birth weight, increased incidence of human papilloma and HIV infections, and cervical cancer [2, 10]. The incidence of infection is high in adolescent girls $[5,6,11]$.

Diagnosis of trichomoniasis is made by observing parasite trophozoites using light microscopy, direct florescent antibody testing, latex agglutination, enzyme-linked immunosorbent assays, and molecular polymerase chain reaction techniques. Culture and staining techniques may be used in diagnosis of disease in samples taken from vaginal, urethral, prostatic secretions, and urine $[12,13]$. Culture is considered to be the criterion standard for diagnosis of trichomoniasis with a sensitivity of 95\% [12]. We performed microscopic examination, Giemsa staining, and CPLM culture on samples taken from the posterior fornix.

Prevalence of $T$. vaginalis was found to be $3.1 \%-$ $8.7 \%$ in reproductive aged women in USA by nucleic acid amplification test $[14,15]$. The prevalence may increase to $38 \%$ in drug using African-American women and $9 \%-30 \%$ in HIV positive women [16, 17]. The prevalence of $T$. vaginalis in Turkish women depends on the study population and diagnostic methods used. Akarsu et al. reported the prevalence of $T$. vaginalis infection among women working in brothels in Ankara at 4.9\% $(n=246)$ where the diagnosis was by direct microscopy and culture [18]. Sonmez-Tamer et al. detected $T$. vaginalis in $5.4 \%$ of women with vaginal discharge by direct microscopy and $9.3 \%$ of these women by culture $(n=128)$ [19]. In a retrospective study, the presence of $T$. vaginalis was investigated in random infertile women with vaginal discharge and in asymptomatic infertile women 2 weeks before in vitro fertilization. Trichomonas vaginalis was detected in only $3 \%$ of women with vaginal discharge using a FDA-cleared nucleic acid probe test [10]. In the present study, we found the prevalence of $\mathrm{T}$. vaginalis was $18 \%$ in infertile women by culture in those with vaginal discharge. Consistent with our study, El-Shazly et al. [20] showed significantly higher T. vaginalis in a group of infertile women compared with a control group (14.58\% vs $2.5 \%$ respectively).

Increased levels of IgA, IgG, Th1 cytokines and reactive nitrogen intermediates were observed in rats having experimentally induced $T$. vaginalis $[8,21]$. Antibodies against $T$. vaginalis were detected in circulation and vaginal mucosa of infected women $[8,22,23]$. T. vaginalis cysteine proteases including CP30 may induce apoptosis of vaginal epithelial cells and multiple mucosal immune cell types [8, 24]. These immunological changes may be a cause of infertility. 
The prevalence of $T$. vaginalis infection increased in infertile women having tubal factor infertility and pelvic inflammatory disease [20, 25]. Interestingly, the prevalence of $T$. vaginalis infection was $25 \%$ in women with male factor infertility and $75 \%$ in women with unexplained infertility. T. vaginalis was not detected in women with tubal factor infertility in our study. These findings may be related to immunological changes because of infection or independently. Our study is limited because of its small sample size.

\section{Conclusion}

Asymptomatic infertile women should be examined for $T$. vaginalis infection. Further studies are needed to elucidate the pathophysiology of infertility in relation to $T$. vaginalis infection.

\section{Acknowledgments}

We thank to Dr. Mutlu Yar, Inonu University, Faculty of Medicine, Department of Parasitology.

\section{Conflict of interest statement}

The authors have no conflicts of interest to declare.

\section{References}

1. Mayta H, Gilman RH, Calderon MM, Gottlieb A, Soto G, Tuero I, et al. 18S Ribosomal DNA-based PCR for diagnosis of Trichomonas vaginalis. J Clin Microbiol. 2000; 38:2683-7.

2. McClelland RS, Sangare L, Hassan WM, Lavreys L, Mandaliya K, Kiarie J, et al. Infection with Trichomonas vaginalis increases the risk of HIV-1 acquisition. J Infect Dis. 2007; 195:698-702.

3. Smith LM, Wang M, Zangwill K, Yeh S. Trichomonas vaginalis infection in a premature newborn. J Perinatol. 2002: 22:502-3.

4. Nijhawan AE, Chapin KC, Salloway R, Andrea S, Champion J, Roberts M, et al. Prevalence and predictors of trichomonas infection in newly incarcerated women. Sex Transm Dis. 2012: 39:973-8.

5. McClelland RS. Trichomonas vaginalis infection: can we afford to do nothing? J Infect Dis. 2008; 197: 487-9.

6. Weinstock H, Berman S, Cates W Jr. Sexually transmitted diseases among American youth: incidence and prevalence estimates, 2000. Perspect Sex Reprod Health. 2004; 36:6-10.

7. Nye MB, Schwebke JR, Body BA. Comparison of
APTIMA Trichomonas vaginalis transcriptionmediated amplification to wet mount microscopy, culture, and polymerase chain reaction for diagnosis of trichomoniasis in men and women. Am J Obstet Gynecol. 2009; 200:181-8.

8. Fichorova RN. Impact of T. vaginalis infection on innate immune responses and reproductive outcome. J Reprod Immunol. 2009; 83:185-9.

9. Kurth A, Whittington WL, Golden MR, Thomas KK, Holmes KK, Schwebke JR. Performance of a new, rapid assay for detection of Trichomonas vaginalis. J Clin Microbiol. 2004: 42:2940-3.

10. Casari E, Ferrario A, Morenghi E, Montanelli A. Trichomonas vaginalis, Candida, Chlamydia trachomatis, Mycoplasma hominis and Ureaplasma urealyticum in the genital discharge of symptomatic fertile and asymptomatic infertile women. New Microbiol. 2010: 33:69-76.

11. Van Der Pol B, Williams JA, Orr DP, Batteiger BE, Fortenberry JD. Prevalence, incidence, natural history, and response to treatment of Trichomonas vaginalis infection among adolescent women. J Infect Dis. 2005; 192:2039-44.

12. Lawing LF, Hedges SR, Schwebke JR. Detection of trichomonosis in vaginal and urine specimens from women by culture and PCR. J Clin Microbiol. 2000; 38: 3585-8.

13. Shafir SC, Sorvillo FJ, Smith L. Current issues and considerations regarding trichomoniasis and human immunodeficiency virus in African-Americans. Clin Microbiol Rev. 2009; 22:37-45.

14. Sutton M, Sternberg M, Koumans EH, McQuillan G, Berman S, Markowitz L. The prevalence of Trichomonas vaginalis infection among reproductiveage women in the United States, 2001-2004. Clin Infect Dis. 2007; 45:1319-26.

15. Ginocchio CC, Chapin K, Smith JS, Aslanzadeh J, Snook J, Hill CS, et al. Prevalence of Trichomonas vaginalis and coinfection with Chlamydia trachomatis and Neisseria gonorrhoeae in the United States as determined by the Aptima Trichomonas vaginalis nucleic acid amplification assay. J Clin Microbiol. 2012; 50:2601-8.

16. Miller M, Liao Y, Gomez AM, Gaydos CA, D’Mellow D. Factors associated with the prevalence and incidence of Trichomonas vaginalis infection among African American women in New York City who use drugs. J Infect Dis. 2008; 197:503-9.

17. Cu-Uvin S, Ko H, Jamieson DJ, Hogan JW, Schuman $\mathrm{P}$, Anderson J, et al. Prevalence, incidence, and 
persistence or recurrence of trichomoniasis among human immunodeficiency virus (HIV)-positive women and among HIV negative women at high risk for HIV infection. Clin Infect Dis. 2002; 34:1406-11.

18. Akarsu GA, elik $\mathrm{T}$, $\mathrm{G}$ ng $\mathrm{r}$, Altinta $\mathrm{K}$. Ankara'da ali an genelev kadinlarinda Trichomonas vaginalis sikli $\mathrm{i}$. [The rate of Trichomonas vaginalis among prostitutes in Ankara]. T rkiye Parazitoloji Dergisi. 2003; 27:252-4. [in Turkish, English abstract]

19. TamerGS,D ndarD, ali kan S, Dog̈erE. Trichomonas vaginalis saptanmasinda direkt mikroskopi ile invitrok lt $r$ nkar ila tirilmasi. [Comparison of direct microscopy and in-vitro cultures in detection of Trichomonas vaginalis]. Turk Hij Den Biyol Derg. 2008; 65:75-80. [in Turkish, English abstract]

20. El-Shazly AM, Al-Naggar HM, Soliman M, El-Negeri M, El-Nemr HE, Handousa AE, et al. A study on trichomoniasis and female infertility. J Egypt Soc Parasitol. 2001; 31:545-53.

21. Malla N, Yadav M, Gupta I. Kinetics of serum and local cytokine profile in experimental intravaginal trichomoniasis induced with Trichomonas vaginalis isolates from symptomatic and asymptomatic women. Parasite Immunol. 2007; 29:101-5.

22. Kaur S, Khurana S, Bagga R, Wanchu A, Malla N. Antitrichomonas IgG, IgM, IgA, and IgG subclass responses in human intravaginal trichomoniasis. Parasitol Res. 2008; 103:305-12.

23. Yadav M, Dubey ML, Gupta I, Malla N. Cysteine proteinase 30 (CP30) and antibody response to CP30 in serum and vaginal washes of symptomatic and asymptomatic Trichomonas vaginalis-infected women. Parasite Immunol. 2007; 29:359-65.

24. Sommer U, Costello CE, Hayes GR, Beach DH, Gilbert RO, Lucas JJ, et al. Identification of Trichomonas vaginalis cysteine proteases that induce apoptosis in human vaginal epithelial cells. J Biol Chem. 2005; 280:23853-60.

25. Klinger EV, Kapiga SH, Sam NE, Aboud S, Chen CY, Ballard RC, et al. A Community-based study of risk factors for Trichomonas vaginalis infection among women and their male partners in Moshi urban district, northern Tanzania. Sex Transm Dis. 2006; 33:712-8. 